\title{
The best sextic approximation of hyperbola with order twelve
}

\author{
Abedallah Rababah ${ }^{1}$, Esra'a Rababah ${ }^{2}$ \\ ${ }^{1}$ Department of Mathematical Sciences, United Arab Emirates University, Al Ain, UAE \\ ${ }^{1,2}$ Department of Mathematics, Jordan University of Science and Technology, Jordan
}

\begin{tabular}{ll}
\hline \hline Article Info & ABSTRACT \\
\cline { 1 - 2 } Article history: & $\begin{array}{l}\text { In this article, the best uniform approximation for the hyperbola of degree } 6 \text { that } \\
\text { has approximation order } 12 \text { is found. The associated error function vanishes } 12\end{array}$ \\
Received Apr 8, 2019 & $\begin{array}{l}\text { times and equioscillates } 13 \text { times. For an arc of the hyperbola, the error is bounded } \\
\text { Revised Oct 20, 2019 }\end{array}$ \\
$\begin{array}{l}\text { Accepted Nov 2, } 2.4 \times 10^{-4} \text {. We explain the details of the derivation and show how to apply } \\
\text { the method. The method is simple and this encourages and motivates people work- } \\
\text { ing in CG and CAD to apply it in their works. }\end{array}$
\end{tabular}

approximation order

equioscillation

Copyright (c) 2020 Insitute of Advanced Engineeering and Science.

high accuracy

All rights reserved.

hyperbola

sextic parametric

approximation

\section{Corresponding Author:}

Abedallah Rababah,

Department of Mathematical Sciences,

United Arab Emirates University,

Al Ain, UAE.

Department of Mathematics,

22110 Irbid, Jordan.

Email: rababah@uaeu.ac.ae and esrababah15@sci.just.edu.jo

\section{INTRODUCTION}

Bézier curves were invented by Pierre Bézier and Paul de Casteljau in the 1960's. The primary application was in the automobile industry, but today they are widely used in many computer applications and codes to sketch curves. Approximation using polynomials of low degree is favourable, and in many cases is a must issue. Many CAD systems are limited to using only parametric polynomial curves of low degree with low errors. The big error causes two major disadvantages: high accumulated error, and slow and costly software. So, it is favourable to approximate using low degree polynomials. Parabolas are represented exactly using parametric curves of degree 2. In [1] methods of best uniform approximation of a circular arc of degree 6 with order 12 are accomplished. These approximations are optimal and have 13 equioscillations. In [2], a method of best uniform approximation of a hyperbola of degrees 2 with order 4 is accomplished. These results were motivated by the results of approximating curves with high accuracy [3-6].

In this paper, we find approximation for the hyperbola of degree 6 and we get the order 12 . Bézier curve techniques are used to represent the approximations of the hyperbola using parametric polynomials of degree 6 that have the least uniform error. The method is simple and practical. To achieve this approximation, proper arrangements and symmetries of the hyperbola are applied to determine the Bézier points that define the approximation of the hyperbola, and therefore, makes a CAD system more efficient and minimizes the cost. Using suitable translation and scaling, the hyperbola can be written in the basic forms: $y^{2}-x^{2}=1$ and $x^{2}-y^{2}=1$. Every form has two branches. So, there are four branches. Geometrically, all of these branches 
are identical. Therefore, it is sufficient to represent one branch and the other three branches can be represented using rotation of this branch. So, we consider the upper branch of the hyperbola $y^{2}-x^{2}=1$, see Figure 1 . It can be written in parametric form $c: t \mapsto(\sinh (t), \cosh (t)), t \in \Re$. We are interested in finding out the longest arc of the hyperbola that can be approximated and that the error function is the Chebyshev polynomial, see [2]. It is impossible to exactly represent a hyperbola with a polynomial curve [7-11]. It can be represented exactly using rational Bézier curves, a polynomial parametric form is preferred in many applications. The ability to represent a primitive hyperbola is a must issue especially in computer graphics and data and image processing. Thus, there is a demand to find a parametrically defined polynomial curve $p_{6}: t \mapsto\left(x_{6}(t), y_{6}(t)\right), 0 \leq t \leq 1$, where $x_{6}(t), y_{6}(t)$ are polynomials of degree 6 . The $p_{6}$ has to approximate $c$ within tolerable error. In this paper, degree 6 parametric curves are considered, and it is shown that the error is very small and the results are competitive.

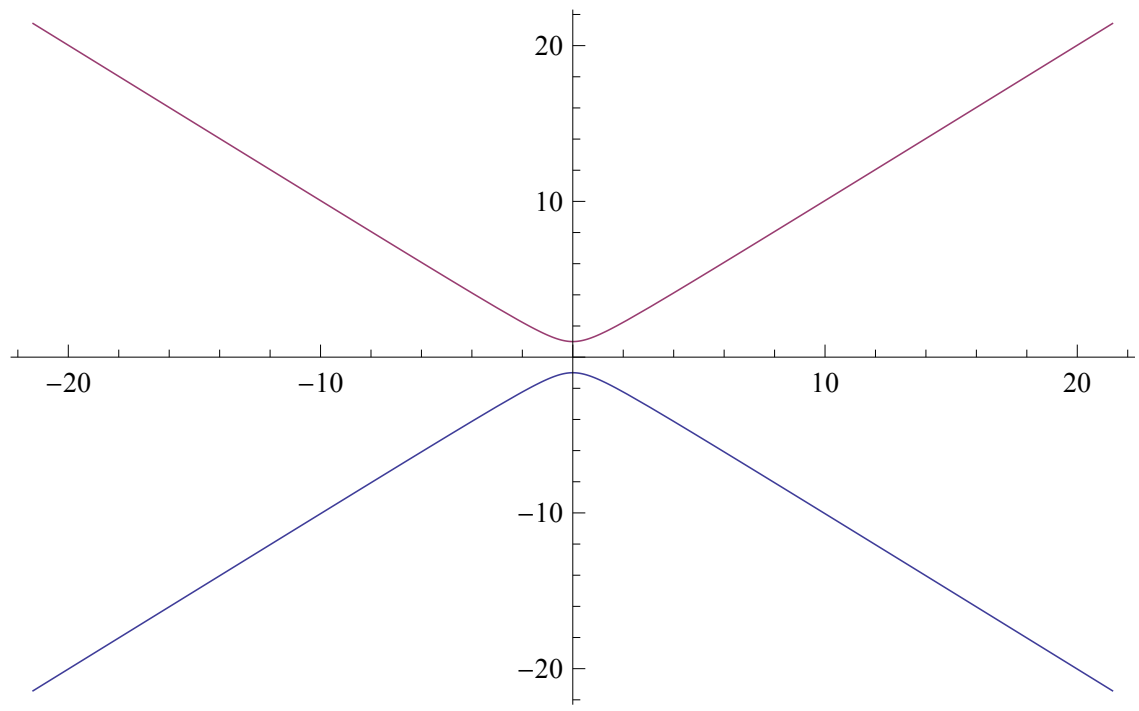

Figure 1. The Hyperbola $y^{2}-x^{2}=1$

The Euclidean error function is used to measure the error between $p_{6}$ and $c$. The error is defined by :

$$
E(t):=\sqrt{y_{6}^{2}(t)-x_{6}^{2}(t)}-1
$$

$E(t)$ is replaced by the following error function:

$$
e(t):=y_{6}^{2}(t)-x_{6}^{2}(t)-1
$$

The approximation problem is formulated as follows.

The approximation problem is to find $p_{6}: t \mapsto\left(x_{6}(t), y_{6}(t)\right), 0 \leq t \leq 1$, where $x_{6}(t), y_{6}(t)$ are polynomials of degree 6 , that approximates $c$ by satisfying the following three conditions:

(a) $p_{6}$ minimizes $\max _{t \in[0,1]}|e(t)|$,

(b) $p_{6}$ approximates $c$ with order twelve [12],

(c) $e(t)$ equioscillates thirteen times over $[0,1]$.

The solution to this problem is shown in section 3. This solution is presented in Figure 2 and Figure 3; the corresponding error is shown in Figure 4. More related results can be found in [13-15] and the references therein. 


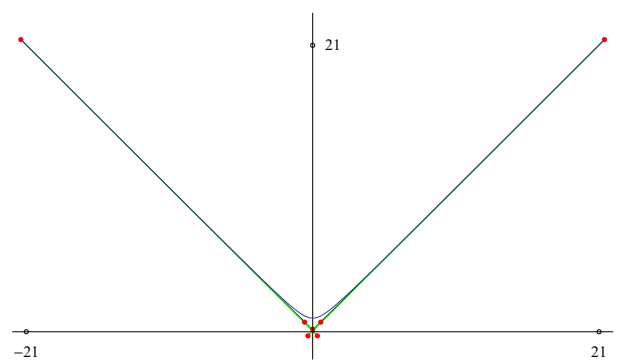

Figure 2. The Hyperbola and the sextic approximating Bézier curve

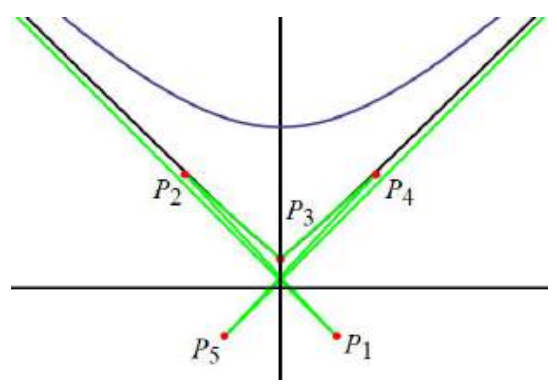

Figure 3. Zoom in the busy part of Figure 2

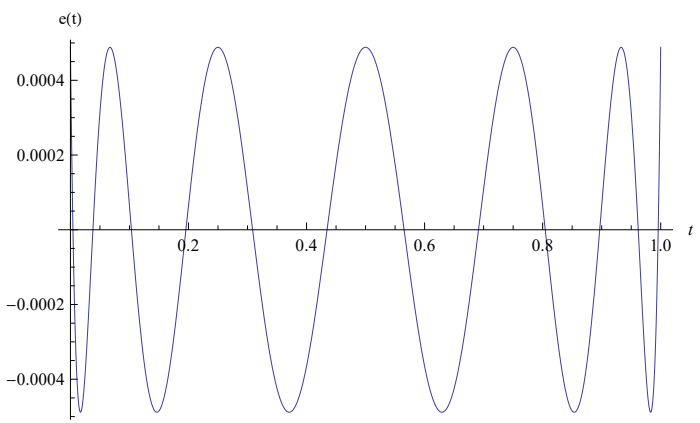

Figure 4. The error of the sextic approximating Bézier curve

\section{RESEARCH METHOD}

Let $p_{6}(t)=\left(x_{6}(t), y_{6}(t)\right)$ be a sextic polynomial parametric representation of the curve $c$. In CAGD, curves are presented using the Bézier form, see Figure 5 for possible choice of the Bézier points.

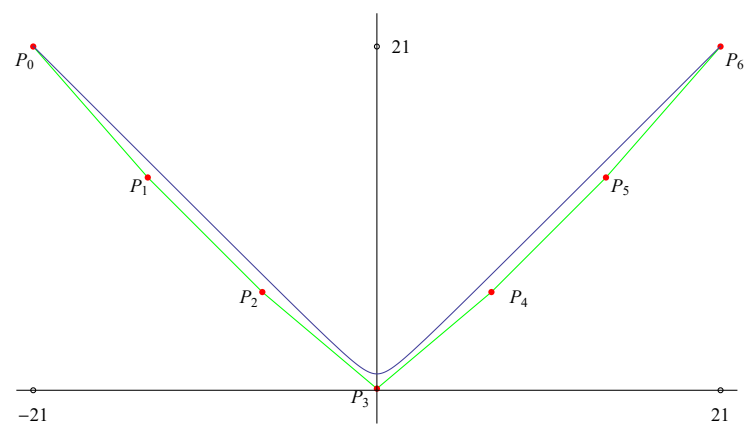

Figure 5. Possible Bézier points of the hyperbola

The Bézier curve $p_{6}(t)$ has the following form:

$$
p_{6}(t)=\sum_{i=0}^{6} p_{i} B_{i}^{6}(t)=:\left(\begin{array}{l}
x_{6}(t) \\
y_{6}(t)
\end{array}\right), \quad 0 \leq t \leq 1,
$$

where $p_{0}, p_{1}, p_{2}, p_{3}, p_{4}, p_{5}, p_{6}$ are the Bézier points, and the Bernstein polynomial basis of degree 6 is defined by:

$$
B_{i}^{6}(t)=\left(\begin{array}{l}
6 \\
i
\end{array}\right)(1-t)^{6-i} t^{i}, \quad i=0, \ldots, 6, \quad t \in[0,1]
$$


The simplicity of this method should encourage people working in the fields of Computer Graphics, Image Processing, CAD, and Data Processing to adopt it in their design and applications. The symmetry in the hyperbola is used to better locate the Bézier points. We begin by letting $p_{0}=(-\alpha, \beta)$. The hyperbola is symmetric around the $y$-axis, so, to obey this symmetry, the point $p_{6}$ should have the form $p_{6}=(\alpha, \beta)$. The point $p_{1}=(-\gamma, \delta)$, so, to obey the symmetry, the point $p_{5}$ should have the form $p_{5}=(\gamma, \delta)$. The point $p_{2}=(-\xi, \psi)$, so, to obey the symmetry, the point $p_{4}$ should have the form $p_{4}=(\xi, \psi)$. There is one remaining point; if this point lies in either halves of the plane around the $y$-axis, then the symmetry of the hyperbola is kicked. Thus, the point $p_{3}$ must lie on the $y$-axis and has the form $p_{3}=(0, \omega)$. Therefore, the proper choice for the Bézier points is

$p_{0}=\left(\begin{array}{c}-\alpha \\ \beta\end{array}\right), p_{1}=\left(\begin{array}{c}-\gamma \\ \delta\end{array}\right), p_{2}=\left(\begin{array}{c}-\xi \\ \psi\end{array}\right), p_{3}=\left(\begin{array}{c}0 \\ \omega\end{array}\right), p_{4}=\left(\begin{array}{c}\xi \\ \psi\end{array}\right), p_{5}=\left(\begin{array}{c}\gamma \\ \delta\end{array}\right), p_{6}=\left(\begin{array}{c}\alpha \\ \beta\end{array}\right)$.

The Bézier polynomial curve $p_{6}(t)$ in $(3)$ is given in the form

$$
\left(\begin{array}{c}
x_{6}(t) \\
y_{6}(t)
\end{array}\right)=\left(\begin{array}{c}
-\alpha B_{0}^{6}(t)-\gamma B_{1}^{6}(t)-\xi B_{2}^{6}(t)+\xi B_{4}^{6}(t)+\gamma B_{5}^{6}(t)+\alpha B_{6}^{6}(t) \\
\beta B_{0}^{6}(t)+\delta B_{1}^{6}(t)+\psi B_{2}^{6}(t)+\omega B_{3}^{6}(t)+\psi B_{4}^{6}(t)+\delta B_{5}^{6}(t)+\beta B_{6}^{6}(t)
\end{array}\right),
$$

where $0 \leq t \leq 1$. The seven parameters $\alpha, \beta, \gamma, \delta, \xi, \psi, \omega$ are used to have the polynomial approximation $p_{6}$ comply with the conditions of the approximation problem; this is done in the following section.

\section{RESULTS AND ANALYSIS}

The values of $\alpha, \beta, \gamma, \delta, \xi, \psi, \omega$ that minimize the uniform error and satisfy the conditions of the approximation problem are given in the following theorem. A symbolic programming language is used to get the values of the parameters in (4) and are rounded in decimal form.

Theorem 1: The Bézier curve in (5) with the Bézier points in (4), where

$$
\begin{aligned}
\alpha & =21.396696163346007, \beta=21.420062908119476, \gamma=-0.34812943434024657 \\
\delta & =-0.3015887594987887, \xi=0.5937616113806532, \psi=0.705589524343983 \\
\omega & =0.1813438330271954
\end{aligned}
$$

satisfies the three conditions of the Approximation Problem. More precisely, the error functions satisfy:

$$
-\frac{1}{2^{11}} \leq e(t) \leq \frac{1}{2^{11}}, \quad-\frac{1}{2^{11}(2-\epsilon)} \leq E(t) \leq \frac{1}{2^{11}(2+\epsilon)}, \text { where } \epsilon=\max _{0 \leq t \leq 1}|E(t)|
$$

Proof: From equation (5), we get

$$
\begin{aligned}
& x_{6}(t)=\alpha\left(B_{6}^{6}(t)-B_{0}^{6}(t)\right)+\gamma\left(B_{5}^{6}(t)-B_{1}^{6}(t)\right)+\xi\left(B_{4}^{6}(t)-B_{2}^{6}(t)\right), \\
& y_{6}(t)=\beta\left(B_{6}^{6}(t)+B_{0}^{6}(t)\right)+\delta\left(B_{5}^{6}(t)+B_{1}^{6}(t)\right)+\psi\left(B_{4}^{6}(t)+B_{2}^{6}(t)\right)+\omega B_{3}^{6}(t) .
\end{aligned}
$$

Substituting $x_{6}(t)$ and $y_{6}(t)$ into the error function $e(t)$ in (2) and rewriting the result in terms of powers of $t$ we get the following equality:

$$
\begin{aligned}
e(t)= & t^{12}\left(4 \beta^{2}-48 \beta \delta+144 \delta^{2}+120 \beta \xi-720 \delta \xi+900 \xi^{2}-80 \beta \omega+480 \delta \omega-1200 \xi \omega+400 \omega^{2}\right) \\
+t^{11}( & \left.-24 \beta^{2}+288 \beta \delta-864 \delta^{2}-720 \beta \xi+4320 \delta \xi-5400 \xi^{2}+480 \beta \omega-2880 \delta \omega+7200 \xi \omega-2400 \omega^{2}\right) \\
& +t^{10}\left(-36 \alpha^{2}+288 \alpha \gamma-576 \gamma^{2}-360 \alpha \xi+1440 \gamma \xi-900 \xi^{2}+96 \beta^{2}-1032 \beta \delta+2736 \delta^{2}\right. \\
& \left.\quad+2400 \beta \psi-12600 \delta \psi+14400 \psi^{2}-1560 \beta \omega+8160 \delta \omega-18600 \psi \omega+6000 \omega^{2}\right) \\
+ & t^{9}\left(180 \alpha^{2}-1440 \alpha \gamma+2880 \gamma^{2}+1800 \alpha \xi-7200 \gamma \xi+4500 \xi^{2}-260 \beta^{2}+2520 \beta \delta-5760 \delta^{2}\right.
\end{aligned}
$$




$$
\begin{aligned}
& \left.-5400 \beta \psi+23400 \delta \psi-22500 \psi^{2}+3400 \beta \omega-14400 \delta \omega+27000 \psi \omega-8000 \omega^{2}\right) \\
& +t^{8}\left(-465 \alpha^{2}+3480 \alpha \gamma-6480 \gamma^{2}-4170 \alpha \xi+15480 \gamma \xi-9225 \xi^{2}+525 \beta^{2}-4440 \beta \delta\right. \\
& \left.+8640 \delta^{2}+8430 \beta \psi-29880 \delta \psi+22725 \psi^{2}-5040 \beta \omega+17040 \delta \omega-24000 \psi \omega+6000 \omega^{2}\right) \\
& +t^{7}\left(780 \alpha^{2}-5280 \alpha \gamma+8640 \gamma^{2}+5880 \alpha \xi-18720 \gamma \xi+9900 \xi^{2}-804 \beta^{2}+5808 \beta \delta-9504 \delta^{2}\right. \\
& \left.-9240 \beta \psi+26640 \delta \psi-15300 \psi^{2}+5040 \beta \omega-13440 \delta \omega+13200 \psi \omega-2400 \omega^{2}\right) \\
& +t^{6}\left(-922 \alpha^{2}+5460 \alpha \gamma-7488 \gamma^{2}-5460 \alpha \xi+13860 \gamma \xi-5850 \xi^{2}+926 \beta^{2}-5628 \beta \delta\right. \\
& \left.+7632 \delta^{2}+7140 \beta \psi-16380 \delta \psi+6750 \psi^{2}-3360 \beta \omega+6720 \delta \omega-4200 \psi \omega+400 \omega^{2}\right) \\
& +t^{5}\left(792 \alpha^{2}-3948 \alpha \gamma+4320 \gamma^{2}+3360 \alpha \xi-6300 \gamma \xi+1800 \xi^{2}-792 \beta^{2}+3972 \beta \delta-4320 \delta^{2}\right. \\
& \left.-3840 \beta \psi+6660 \delta \psi-1800 \psi^{2}+1440 \beta \omega-1920 \delta \omega+600 \psi \omega\right) \\
& +t^{4}\left(-495 \alpha^{2}+1980 \alpha \gamma-1620 \gamma^{2}-1320 \alpha \xi+1620 \gamma \xi-225 \xi^{2}+495 \beta^{2}-1980 \beta \delta+1620 \delta^{2}\right. \\
& \left.+1380 \beta \psi-1620 \delta \psi+225 \psi^{2}-360 \beta \omega+240 \delta \omega\right) \\
& +t^{3}\left(220 \alpha^{2}-660 \alpha \gamma+360 \gamma^{2}+300 \alpha \xi-180 \gamma \xi-220 \beta^{2}+660 \beta \delta-360 \delta^{2}-300 \beta \psi\right. \\
& +180 \delta \psi+40 \beta \omega) \\
& +t^{2}\left(-66 \alpha^{2}+132 \alpha \gamma-36 \gamma^{2}-30 \alpha \xi+66 \beta^{2}-132 \beta \delta+36 \delta^{2}+30 \beta \psi\right) \\
& +t\left(12 \alpha^{2}-12 \alpha \gamma-12 \beta^{2}+12 \beta \delta\right)+\left(-1-\alpha^{2}+\beta^{2}\right) .
\end{aligned}
$$

We substitute the values of $\alpha, \beta, \gamma, \delta, \xi, \psi$ and $\omega$ from (6) into the last equation to get

$$
\begin{gathered}
e(t)=4096 t^{12}-24576 t^{11}+64512 t^{10}-97280 t^{9}+93024 t^{8}-58752 t^{7}+24752 t^{6} \\
-6864 t^{5}+\frac{19305}{16} t^{4}-\frac{1001}{8} t^{3}+\frac{429}{64} t^{2}-\frac{9}{64} t+\frac{1}{2048}, \quad t \in[0,1] .
\end{gathered}
$$

Making the substitution $t=\frac{u+1}{2}$ reduces the error function to the following form

$$
e(u)=\frac{1}{2048}-\frac{9}{256} u^{2}+\frac{105}{256} u^{4}-\frac{7}{4} u^{6}+\frac{27}{8} u^{8}+3 t^{10}+t^{12}, \quad u \in[-1,1] .
$$

The last form of $e(u)$ coincides with the monic Chebyshev polynomial $\tilde{T}_{12}(u), u \in[-1,1]$, which is the unique polynomial of degree 12 that equioscillates 13 times between $\pm \frac{1}{2^{11}}$ for all $u \in[-1,1]$ and has the least deviation [12]. This shows that $p_{6}$ satisfies the conditions of the approximation problem. The error formula for $E(t)$ can be proved using its relation to the error function $e(t)$. This proves Theorem 1 .

It is clear that the approximation is the best uniform approximation from Figure 4. Figure 2 and Figure 3 show the hyperbola and the approximating Bézier curve, Figure 4 shows the corresponding error, and Figure 6 shows the Euclidean error.

\section{CONCLUSION}

In this paper, the best uniform approximation of the hyperbola with parametrically defined polynomial curves of degree 6 are explicitly given. The error function equioscillates 13 times; the approximation order is 12 . The method of construction demonstrates the efficiency and simplicity of the approximation method. The approximation intersects the hyperbola 12 times with maximum error $2.4 \times 10^{-4}$. Reflecting the upper branch of the hyperbola around the $x$-axis gives the lower branch. The hyperbola is shown in Figure 7. Note that the points $p_{1}\left(p_{5}\right)$ and $p_{2}\left(p_{4}\right)$ in both branches are very close to each other and can not be distinguished from each other. The results in this paper can be used to improve the results obtained in [16-23] see also the results in $[24,25]$. 


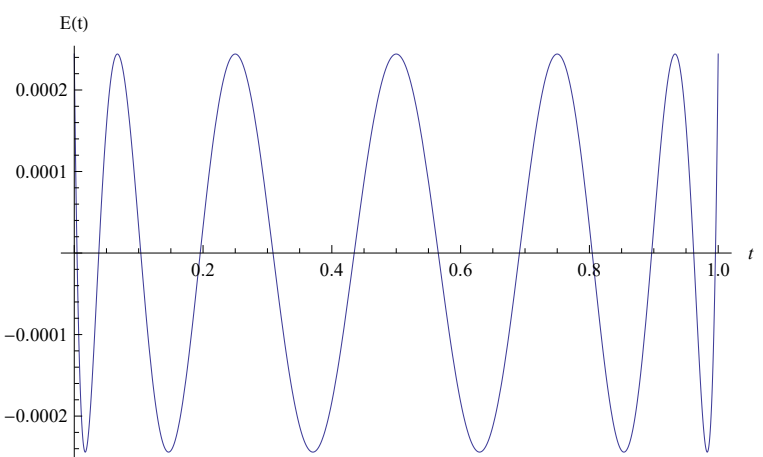

Figure 6. The Euclidean error of the sextic Bézier curve

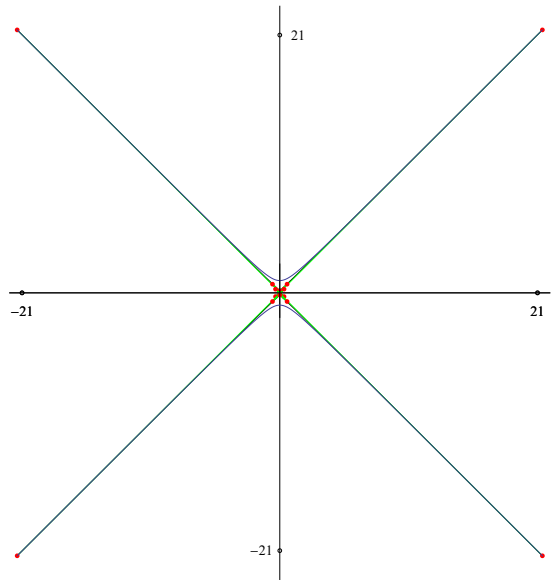

Figure 7. Both branches of the Hyperbola using two Bézier curves

\section{ACKNOWLEDGMENTS}

The authors owe thanks for the reviewers for invaluable suggestions to improve an earlier version of this paper. This research was funded by Jordan University of Science and Technology.

\section{REFERENCES}

[1] A. Rababah, "Best sextic approximation of circular arcs with thirteen equioscillations," Proceedings of the Jangjeon Mathematical Society, vol. 21, Issue 1, pp. 111-123, 2018. DOI: http://doi.org/10.17777/pjms2018.21.1.111

[2] A. Rababah, "The Best Quadratic Approximation of Hyperbola with Order Four," Journal of Inequalities and Special Functions, vol. 7, Issue 3, pp. 1-11, 2016.

[3] M. Floater, "High order approximation of conic sections by quadratic splines," Computer Aided Geometric Design, vol. 12, pp. 617-637, 1995.

[4] C. de Boor., et al., "High accuracy geometric Hermite interpolation," Computer Aided Geometric Design, vol. 4, pp. 269-278, 1988.

[5] A. Rababah, "Taylor theorem for planar curves," Proceedings of the American Mathematical Society, vol. 119, Issue 3, pp. 803-810, 1993.

[6] A. Rababah, "High accuracy Hermite approximation for space curves in $\Re^{d}$," Journal of Mathematical Analysis and Applications, vol. 325, Issue 2, pp. 920-931, 2007.

[7] K. Höllig and J. Hörner, "Approximation and Modeling with B-Splines," SIAM, Titles in Applied Mathematics, vol. 132, 2013.

[8] H. Prautzsch., et al., "Bézier and B-Spline Techniques," Springer 2002. 
[9] P. Bézier, "The mathematical basis of the UNISURF CAD system," Butterworth-Heinemann Newton, MA, USA, ISBN 0-408-22175-5, 1986.

[10] Carl de Boor, A Practical Guide to Splines, Series Title: Applied Mathematical Sciences, Vol 27, SpringerVerlag New York, 1978.

[11] Gerald Farin, Curves and surfaces for computer-aided geometric design: a practical guide. Elsevier, 2014.

[12] J. Rice, "The approximation of functions," linear theory, Addison-Wesley, vol. 1, 1964.

[13] Y. J. Ahn and C. Hoffmann, "Circle approximation using LN Bézier curves of even degree and its application," Journal of Mathematical Analysis and Applications, vol. 410 Issue 1, pp. 257-266, February 2014.

[14] S. W. Kim and Y. J. Ahn, "Circle approximation by quartic $\mathrm{G}^{2}$ spline using alternation of error function," J. KSIAM, vol. 17, Issue 5, pp. 171-179, 2013.

[15] A. Rababah., et al., "Multiple Degree Reduction and Elevation of Bézier Curves Using Jacobi-Bernstein Basis Transformations," Numerical Functional Analysis and Optimization, vol. 28, Issue 9-10, pp. 11791196, 2007.

[16] S. Alabed, "Performance Analysis of Differential Beamforming in Decentralized Networks," International Journal of Electrical and Computer Engineering (IJECE), vol. 8 Issue 3, pp. 1692-1700, 2018. DOI: http://doi.org/10.11591/ijece.v8i3.pp1692-1700

[17] C. Suwannapong and C. Khunboa, "An Approximation Delay between Consecutive Requests for Congestion Control in Unicast CoAP-based Group Communication," International Journal of Electrical and Computer Engineering (IJECE), vol. 9, Issue 3, June 2019. DOI: http://doi.org/10.11591/ijece.v9i3.

[18] Bhargav Bhatkalkar, Abhishek Joshi, Srikanth Prabhu, Sulatha Bhandary, Automated fundus image quality assessment and segmentation of optic disc using convolutional neural networks. International Journal of Electrical and Computer Engineering (IJECE) Vol.10, No.1, 2020, pp. 816 827, ISSN: 2088-8708, DOI: 10.11591/ijece.v10i1.pp816-827.

[19] Rodiah, Sarifuddin Madenda, Diana Tri Susetianigtias, Dewi Agushinta Rahayu, EtySutanty, Optic Disc and Macula Localization from Retinal Optical Coherence Tomography and Fundus Image, International Journal of Electrical and Computer Engineering (IJECE) Vol.8, No.6, 2018, pp. 5050-5060, ISSN: 20888708, DOI: 10.11591/ijece.v8i6.pp5050-5060.

[20] G. Daway, Hana H. kareem, Ahmed Rafid Hashim, Pupil Detection Based on Color Difference and Circular Hough TransformHazim, International Journal of Electrical and Computer Engineering (IJECE) Vol.8, No.5, 2018, pp. 3278-3284, ISSN: 2088-8708, DOI: 10.11591/ijece.v8i5.pp3278-3284.

[21] A. Rababah, L-2 degree reduction of triangular Bézier surfaces with common tangent planes at vertices. International Journal of Computational Geometry \& Applications, Vol. 15, No. 5 (2005) 477-490. http://www.worldscinet.com/ijcga/15/1505/S02181959051505.html

[22] A. Rababah, Distances with rational triangular Bézier surfaces. Applied Mathematics and Computation 160, (2005) 379-386. http://www.elsevier.com/locate/amc

[23] A. Rababah and Moath Jaradat, Approximating offset Curves using Bézier curves with high accuracy, International Journal of Electrical and Computer Engineering (IJECE) Vol.10, No.2, 2020 (PART I).

[24] A. Rababah, "Approximation von Kurven mit Polynomen und Splines," Ph. Dissertation, Stuttgart Universität, West Germany, 1992.

[25] A. Rababah, The best quintic Chebyshev approximation of circular arcs of order ten, International Journal of Electrical and Computer Engineering (IJECE)Vol. 9, No. 5, October 2019, pp. 3779-3785, ISSN: 2088 8708, DOI: 10.11591/ijece.v9i5.pp3779-3785. 


\section{BIOGRAPHIES OF AUTHORS}

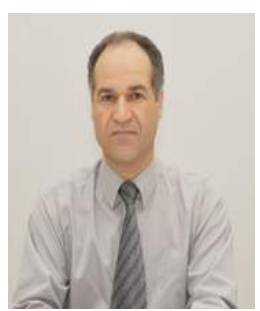

Abedallah Rababah is a professor of mathematics at United Arab Emirates University and is on leave from Jordan University of Science and Technology. He is working in the field of Computer Aided Geometric Design, abbreviated CAGD. In particular, his research is on degree raising and reduction of Bézier curves and surfaces with geometric boundary conditions, Bernstein polynomials, and their duality. He is known for his research in describing approximation methods that significantly improve the standard rates obtained by classical (local Taylor, Hermite) methods. He proved the following conjecture for a particular set of curves of nonzero measure: Conjecture: A smooth regular planar curve can, in general, be approximated by a polynomial curve of degree $n$ with order $2 \mathrm{n}$. The method exploited the freedom in the choice of the parametrization and achieved the order $4 \mathrm{n} / 3$, rather than $n+1$. Generalizations were also proved for space curves. Professor Rababah is also doing research in the fields of classical approximation theory, orthogonal polynomials, Jacobi-weighted orthogonal polynomials on triangular domains, and best uniform approximations. Since 1992, He has been teaching at German, Jordanian, American, Canadian, and Emirates' universities. $\mathrm{He}$ is active in the editorial boards of many journals in mathematics and computer science. Further info can be found on his homepage at ResearchGate: https://www.researchgate.net/profile/Abedallah_Rababah or at http://www.just.edu.jo/eportfolio/Pages/Default.aspx?email=rababah

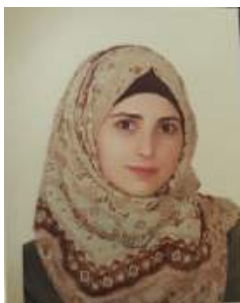

Esra'a Rababah graduated from Jordan University of Science and Technology. She obtained both Bachelor and Master Degrees in Mathematics with research interests in the field of numerical analysis, approximation theory, and Bézier techniques. She worked as teacher assistant at Jordan University of Science and Technology. Further info can be found on her homepage at ResearchGate: https://www.researchgate.net/profile/Esraa_Rababah 